\title{
Asymmetric Multi-channel Sampling in a Series of Shift Invariant Spaces
}

\author{
Adam Zakria \\ University of Kordofan, Faculty of Science, Department of Mathematics, Sudan, adammath2020@gmail.com
}

Received: June 21, 2016 / Accepted: July 22, 2016 / Published: September 25, 2016.

\begin{abstract}
We show asymmetric multi-channel sampling on a series of a shift invariant spaces $\sum_{d=1}^{m} V\left(\varphi\left(t_{d}\right)\right)$ with a series of Riesz generators $\sum_{d=1}^{m} \varphi\left(t_{d}\right)$ in $L^{2}(\mathbb{R})$, where each channeled signal is assigned a uniform but distinct sampling rate. We use Fourier duality between $\sum_{d=1}^{m} V\left(\varphi\left(t_{d}\right)\right)$ and $L^{2}[0,2 \pi]$ to find conditions under which there is a stable asymmetric multi-channel sampling formula on $\sum_{d=1}^{m} V\left(\varphi\left(t_{d}\right)\right)$.
\end{abstract}

Keywords: Shift invariant space, Multi-channel sampling, Frame Riesz basis

\section{Introduction}

The multi-channel sampling method goes back to the works of Shannon [18] and Fogel [7], where reconstruction of a band-limited signal from samples of the signal and its derivatives was found. Generalized sampling expansion using arbitrary multi-channel sampling on the Paley-Wiener space was introduced first by Papoulis [16]. Since Papoulis' fundamental work, there have been many generalizations and applications of multi-channel sampling. See [1,5,6,14,17,19].

Papoulis' result has also been extended to a general shift invariant space by using the filter banks technique (see [4,19,20]). More recently Garcia and Pérez-Villalon [8] derived stable generalized sampling in a shift invariant space. Most previous work related to multi-channel sampling has assumed that the sampling rates of all channels are the same.

S. Kang , J.M. Kim, K.H. Kwon [22] considered asymmetric multi-channel sampling in a shift invariant space $V(\varphi)$ with a suitable Riesz generator $\varphi(t)$, where each channeled signal is sampled with a

Corresponding author: Adam Zakria, University of Kordofan , Faculty of Science, Department of Mathematics, Sudan. E-mail: adammath2020@gmail.com uniform but distinct rate. Using Fourier duality between $\sum_{d=1}^{m} V\left(\varphi\left(t_{d}\right)\right)$ and $L^{2}[0,2 \pi][8,9,10,22]$, we derive under the same considerations a stable series of shifted asymmetric multi-channel sampling formula in $\sum_{d=1}^{m} V\left(\varphi\left(t_{d}\right)\right)$. The corresponding symmetric multi-channel sampling in $\sum_{d=1}^{m} V\left(\varphi\left(t_{d}\right)\right)$ was handled in [9],[22], where $\sum_{d=1}^{m} \varphi\left(t_{d}\right)$ is a continuous series of Riesz generators satisfying

$\sup _{\mathbb{R}} \sum_{n \in \mathbb{Z}} \sum_{d=1}^{m}\left|\varphi\left(t_{d}-n\right)\right|^{2}<\infty$. In this case all signals in $\sum_{d=1}^{m} V\left(\varphi\left(t_{d}\right)\right)$ are continuous on $\mathbb{R}$ [21],[22]. We require only that the series of Riesz generators $\sum_{d=1}^{m} \varphi\left(t_{d}\right)$ are pointwise well defined everywhere on $\mathbb{R}$ and $\sum_{n \in \mathbb{Z}} \sum_{d=1}^{m}\left|\varphi\left(t_{d}-n\right)\right|^{2}<$ $\infty, \sum_{d=1}^{m} t_{d} \in \mathbb{R}$. Hence we essentially allow any series of Riesz generators in $L^{2}(\mathbb{R})$. Hence [22] allow more general filters than the ones in [8] by asking only that the impulse responses of filters belong to $L^{2}(\mathbb{R})$ (or the frequency responses of filters belong to $L^{2}(\mathbb{R}) \cup L^{\infty}(\mathbb{R})$ when $\sum_{n \in \mathbb{Z}}|\hat{\varphi}(\xi+2 n \pi)| \in$ $\left.L^{2}[0,2 \pi]\right)$, whereas they belong to $L^{2}(\mathbb{R}) \cap L^{1}(\mathbb{R})$ in [9]. We give an illustrative examples (see[22]).

\section{Preliminaries}

We consider the notations and formulas in [22]. The 
normalized Fourier transform is

$$
\begin{aligned}
& \mathcal{F}[\varphi](\xi)=\hat{\varphi}(\xi) \\
& =\int_{-\infty}^{\infty} \sum_{d=1}^{m} \varphi\left(t_{d}\right) \prod_{d=1}^{m} e^{-i t_{d} \xi} d t_{d}, \sum_{d=1}^{m} \varphi\left(t_{d}\right) \\
& \in L^{2}(\mathbb{R}) \cap L^{1}(\mathbb{R})
\end{aligned}
$$

so that $\frac{1}{\sqrt{2 \pi}} \mathcal{F}[\cdot]$ extends to a unitary operator from $L^{2}(\mathbb{R})$ onto $L^{2}(\mathbb{R})$. For any $\sum_{d=1}^{m} \varphi\left(t_{d}\right) \in L^{2}(\mathbb{R})$, let

$$
\begin{gathered}
\sum_{d=1}^{m} C_{\varphi}\left(t_{d}\right)=\sum_{n \in \mathbb{Z}} \sum_{d=1}^{m}\left|\varphi\left(t_{d}+n\right)\right|^{2} \text { and } G_{\varphi}(\xi) \\
=\sum_{n \in \mathbb{Z}}|\hat{\varphi}(\xi+2 n \pi)|^{2} .
\end{gathered}
$$

Then

$$
\begin{gathered}
\sum_{d=1}^{m} C_{\varphi}\left(t_{d}\right)=\sum_{d=1}^{m} C_{\varphi}\left(t_{d}+1\right) \in L^{1}[0,1], G_{\varphi}(\xi) \\
=G_{\varphi}(\xi+2 \pi) \in L^{2}[0,2 \pi]
\end{gathered}
$$

and

$$
\begin{gathered}
\left\|\sum_{d=1}^{m} \varphi\left(t_{d}\right)\right\|_{L^{2(\mathbb{R})}}^{2}=\left\|\sum_{d=1}^{m} C_{\varphi}\left(t_{d}\right)\right\|_{L^{1}[0,1]} \\
=\frac{1}{2 \pi}\left\|G_{\varphi}(\xi)\right\|_{L^{1}[0,2 \pi]} .
\end{gathered}
$$

In particular, $\sum_{d=1}^{m} C_{\varphi}\left(t_{d}\right)<\infty$ for a.e. $\sum_{d=1}^{m} t_{d} \in \mathbb{R}$. We also let

$$
\sum_{d=1}^{m} Z_{\varphi}\left(t_{d}, \xi\right)=\sum_{n \in \mathbb{Z}} \sum_{d=1}^{m} \varphi\left(t_{d}+n\right) e^{-i n \xi}
$$

be the Zak transform [12] of $\sum_{d=1}^{m} \varphi\left(t_{d}\right)$ in $L^{2}(\mathbb{R})$ ). Then $\sum_{d=1}^{m} Z_{\varphi}\left(t_{d}, \xi\right)$ is well defined a.e. on $\mathbb{R}^{2}$ and is quasi-periodic in the sense that

$$
\sum_{d=1}^{m} Z_{\varphi}\left(t_{d}+1, \xi\right)=e^{i \xi} \sum_{d=1}^{m} Z_{\varphi}\left(t_{d}, \xi\right)
$$

and

$$
\sum_{d=1}^{m} Z_{\varphi}\left(t_{d}, \xi+2 \pi\right)=\sum_{d=1}^{m} Z_{\varphi}\left(t_{d}, \xi\right) .
$$

A Hilbert space $H$ consisting of complex valued functions on a set $E$ is called a reproducing kernel Hilbert space (RKHS in short) if there is a series of a functions $\sum_{d=1}^{m} q\left(s_{d}, t_{d}\right)$ on $E \times E$, called the reproducing kernel of $H$, satisfying

(i) $\sum_{d=1}^{m} q\left(., t_{d}\right) \in H$ for each $\sum_{d=1}^{m} t_{d} \in E$,

(ii) $\sum_{d=1}^{m}\left\langle f\left(s_{d}\right), q\left(s_{d}, t_{d}\right)\right\rangle=\sum_{d=1}^{m} f\left(t_{d}\right), f \in H$.

In an RKHS $H$, any norm converging sequence also converges uniformly on any subset of $E$, on which $\left\|\sum_{d=1}^{m} q\left(., t_{d}\right)\right\|_{H}^{2}=\sum_{d=1}^{m} q\left(t_{d}, t_{d}\right)$ is bounded.

A sequence $\left\{\varphi_{n}: n \in \mathbb{Z}\right\}$ of vectors in a separable Hilbert space $H$ is

(i) a Bessel sequence with a bound $A+\epsilon: \epsilon>0$ if

$$
\sum_{n \in \mathbb{Z}}\left|\left\langle\varphi, \varphi_{n}\right\rangle\right|^{2} \leq(A+\epsilon)\|\varphi\|^{2}, \varphi \in H, \epsilon>0,
$$

(ii) a frame of $H$ with bounds $A+\epsilon \geq A: \epsilon>0$ if

$$
A\|\varphi\|^{2} \leq \sum_{n \in \mathbb{Z}}\left|\left\langle\varphi, \varphi_{n}\right\rangle\right|^{2} \leq(A+\epsilon)\|\varphi\|^{2}, \varphi \in H, \epsilon
$$$$
>0 \text {, }
$$

(iii) a Riesz basis of $H$ with bounds $A+\epsilon \geq A$ : $\epsilon>0$ if it is complete in $H$ and

$$
\begin{array}{r}
A\|\boldsymbol{c}\|^{2} \leq\left\|\sum_{n \in \mathbb{Z}} c(n) \varphi_{n}\right\|^{2} \leq(A+\epsilon)\|c\|^{2}, c \\
=\{c(n)\}_{n \in \mathbb{Z}} \in l^{2}, \epsilon>0,
\end{array}
$$

where $\|\boldsymbol{c}\|^{2}=\sum_{n \in \mathbb{Z}}|\mathrm{c}(\mathrm{n})|^{2}$.

We let $\sum_{d=1}^{m} V\left(\varphi\left(t_{d}\right)\right)$ be the series of the shift invariant spaces, where $\sum_{d=1}^{m} \varphi\left(t_{d}\right)$ is a series of a Riesz generators, that is, $\left\{\sum_{d=1}^{m} \varphi\left(t_{d}-n\right): n \in \mathbb{Z}\right\}$ is a series of a Riesz bases of $\sum_{d=1}^{m} V\left(\varphi\left(t_{d}\right)\right)$. Then

$$
\begin{aligned}
\sum_{d=1}^{m} V\left(\varphi\left(t_{d}\right)\right)= & \left\{\sum_{d=1}^{m}(\boldsymbol{c} * \varphi)\left(t_{d}\right)\right. \\
& =\sum_{n \in \mathbb{Z}} \sum_{d=1}^{m} c(n) \varphi\left(t_{d}-n\right): C \\
& \left.=\{c(n)\}_{n \in \mathbb{Z}} \in l^{2}\right\} .
\end{aligned}
$$


It is well known see [2] that $\sum_{d=1}^{m} \varphi\left(t_{d}\right)$ is a series of a Riesz generators if and only if there are constant $A$ such that $A \leq G_{\varphi}(\xi) \leq A+\epsilon$ a.e.on $[0,2 \pi]$. In this case, $\left\{\sum_{d=1}^{m} \varphi\left(t_{d}-n\right): n \in \mathbb{Z}\right\}$ is a series of a Riesz bases of $\sum_{d=1}^{m} V\left(\varphi\left(t_{d}\right)\right)$ with bound $\epsilon>0$. We assume further that

(i) $\sum_{d=1}^{m} \varphi\left(t_{d}\right)$ is everywhere well defined on $\mathbb{R}$;

$\sum_{d=1}^{m} C_{\varphi}\left(t_{d}\right)<\infty, \sum_{d=1}^{m} t_{d} \in \mathbb{R}$, i.e.,$\left\{\sum_{d=1}^{m} \varphi\left(t_{d}-\right.\right.$ $n): n \in \mathbb{Z}\} \in l 2$ for each $d=1 m t d \in \mathbb{R}$

We then allow essentially all series of Riesz generators since for any $\sum_{d=1}^{m} \varphi\left(t_{d}\right) \in L^{2}(\mathbb{R}), \sum_{d=1}^{m} C_{\varphi}\left(t_{d}\right)<\infty \quad$ a.e. $\quad$ so that $\sum_{d=1}^{m} \varphi\left(t_{d}\right)$ has an equivalent representative satisfying the above two conditions. Then for each $\boldsymbol{c} \in l^{2}, \sum_{d=1}^{m}(\boldsymbol{c} * \varphi)\left(t_{d}\right)$ converges both in $L^{2}(\mathbb{R})$ and absolutely for each $\sum_{d=1}^{m} t_{d} \in \mathbb{R}$. Hence $\sum_{d=1}^{m} V\left(\varphi\left(t_{d}\right)\right)$ becomes an RKHS with the reproducing kernel (see [13])

$$
\begin{aligned}
& \sum_{d=1}^{m} q\left(s_{d}, t_{d}\right) \\
& =\sum_{n \in \mathbb{Z}} \sum_{d=1}^{m} \tilde{\varphi}\left(s_{d}-n\right) \varphi \overline{\left(t_{d}-n\right)} \text {, where }\left\{\sum _ { d = 1 } ^ { m } \tilde { \varphi } \left(t_{d}\right.\right. \\
& -n): n \in \mathbb{Z}\}
\end{aligned}
$$

is the series of the dual Riesz bases of $\left\{\sum_{d=1}^{m} \varphi\left(t_{d}-\right.\right.$ $n$ ): $n \in \mathbb{Z}\}$ with bounds for $\epsilon>0$. As in $[9,10]$, we introduce an isomorphism $\mathcal{J}$ from $L^{2}[0,2 \pi]$ onto $\sum_{d=1}^{m} V\left(\varphi\left(t_{d}\right)\right)$ defined as:

$$
\begin{aligned}
& \sum_{d=1}^{m}(\mathcal{J} F)\left(t_{d}\right) \\
& =\frac{1}{2 \pi} \sum_{n \in \mathbb{Z}} \sum_{d=1}^{m}\left\langle F(\xi), e^{-i n \xi}\right\rangle_{L^{2}[0,2 \pi]} \varphi\left(t_{d}-n\right) \\
& =\sum_{d=1}^{m}\left\langle F(\xi), \frac{1}{2 \pi} Z_{\varphi}\left(t_{d}, \xi\right)\right\rangle_{L^{2}[0,2 \pi]} .
\end{aligned}
$$

We then have:

(i) $(\widehat{\mathcal{J} F})(\xi)=F(\xi) \hat{\varphi}(\xi)$

(ii) $\mathcal{J}\left(F(\xi) e^{-i n \xi}\right)=\sum_{d=1}^{m}(\mathcal{J} F)\left(t_{d}-n\right), n \in$ $\mathbb{Z}$.

\section{Asymmetric Multi-channel Sampling}

The aim of this paper is as follows (see [22]). Let $\left\{L_{\left(1+\epsilon_{1}\right)}[\cdot]: \epsilon_{1} \geq 0\right\}$ be $N$ LTI (linear time-invariant) systems with impulse responses $\left\{\sum_{d=1}^{m} L_{\left(1+\epsilon_{1}\right)}\left(t_{d}\right): \epsilon_{1} \geq 0\right\}$. Develop a stable series of shifted multi-channel sampling formula for any signal $\sum_{d=1}^{m} f\left(t_{d}\right) \in \sum_{d=1}^{m} V\left(\varphi\left(t_{d}\right)\right) \quad$ using discrete sample values from $\left\{\sum_{d=1}^{m} L_{\left(1+\epsilon_{1}\right)}\left(t_{d}\right): \epsilon_{1} \geq 0\right\}$, where each channeled signal $\sum_{d=1}^{m} L_{\left(1+\epsilon_{1}\right)}[f]\left(t_{d}\right)$ for $\epsilon_{1} \geq 0$ is assigned with a distinct sampling rate

$$
\begin{aligned}
\sum_{d=1}^{m} f\left(t_{d}\right)=\sum_{\epsilon_{1}=0}^{N} & \sum_{n \in \mathbb{Z}} \sum_{d=1}^{m} L_{\left(1+\epsilon_{1}\right)}[f]\left(\sigma_{\left(1+\epsilon_{1}\right)}\right. \\
& \left.+\left(1+\epsilon_{2}\right)_{\left(1+\epsilon_{1}\right)} n\right) s_{d\left(1+\epsilon_{1}\right), n}\left(t_{d}\right),
\end{aligned}
$$

$$
\sum_{d=1}^{m} f\left(t_{d}\right) \in \sum_{d=1}^{m} V\left(\varphi\left(t_{d}\right)\right)
$$

where $\left\{\sum_{d=1}^{m} s_{d\left(1+\epsilon_{1}\right), n}\left(t_{d}\right): \epsilon_{1} \geq 0, n \in \mathbb{Z}\right\} \quad$ is $\quad$ a series of frames or a Riesz basis of $\sum_{d=1}^{m} V\left(\varphi\left(t_{d}\right)\right)$,

$\left\{\left(1+\epsilon_{2}\right)_{\left(1+\epsilon_{1}\right)}: \epsilon_{1} \geq 0\right\}$ are positive integers, and $\left\{\sigma_{\left(1+\epsilon_{1}\right)}: \epsilon_{1} \geq 0\right\}$ are real constants. Note that the series of shifting of sampling instants is unavoidable in some uniform sampling [12] and arises naturally when we allow rational sampling periods in (1). Here, we assume that each $L_{\left(1+\epsilon_{1}\right)}[\cdot]$ is one of the following three types: the impulse response $\sum_{d=1}^{m} l\left(t_{d}\right)$ of an LTI system is such that

(i) $\sum_{d=1}^{m} l\left(t_{d}\right)=\sum_{d=1}^{m} \delta\left(t_{d}+a\right), a \in \mathbb{R}$ or

(ii) $\sum_{d=1}^{m} l\left(t_{d}\right) \in L^{2}(\mathbb{R})$ or

(iii) $\hat{l}(\xi) \in L^{\infty}(\mathbb{R}) \cup L^{2}(\mathbb{R})$ when

$H_{\varphi}(\xi)=\sum_{n \in \mathbb{Z}}|\hat{\varphi}(\xi+2 n \pi)| \in L^{2}[0,2 \pi] \quad$.For type (i),

$$
\sum_{d=1}^{m} L[f]\left(t_{d}\right)=\sum_{d=1}^{m} f\left(t_{d}+a\right), f \in L^{2}(\mathbb{R})
$$
so that $L[\cdot]: L^{2}(\mathbb{R}) \rightarrow L^{2}(\mathbb{R})$ is an isomorphism. In 
particular, for any

$$
\begin{gathered}
\sum_{d=1}^{m} f\left(t_{d}\right)=\sum_{d=1}^{m}(\boldsymbol{c} * \varphi)\left(t_{d}\right) \in \sum_{d=1}^{m} V\left(\varphi\left(t_{d}\right)\right) \\
\sum_{d=1}^{m} L[f]\left(t_{d}\right)=\sum_{d=1}^{m}(\boldsymbol{c} * \psi)\left(t_{d}\right)
\end{gathered}
$$

converges absolutely on $\mathbb{R}$ since

$$
\begin{aligned}
\sum_{d=1}^{m} C_{\psi}\left(t_{d}\right)= & \sum_{n \in \mathbb{Z}} \sum_{d=1}^{m}\left|\psi\left(t_{d}+n\right)\right|^{2}<\infty, \sum_{d=1}^{m} t_{d} \\
\in \in \mathbb{R}, \text { where } & \\
\sum_{d=1}^{m} \psi\left(t_{d}\right) & =\sum_{d}^{m} L[\varphi]\left(t_{d}\right)=\sum_{d=1}^{m} \varphi\left(t_{d}+a\right)
\end{aligned}
$$

For types (ii) and (iii), we have the following results (see [22]):

Lemma 3.1. Let $L[\cdot]$ be an LTI system with the impulse response $\sum_{d=1}^{m} l\left(t_{d}\right)$ of the type (ii) or (iii) as above and

$$
\sum_{d=1}^{m} \psi\left(t_{d}\right)=\sum_{d=1}^{m} L[\varphi]\left(t_{d}\right)=\sum_{d=1}^{1}(\varphi * l)\left(t_{d}\right)
$$

Then

(a) $\sum_{d=1}^{m} \psi\left(t_{d}\right) \in C_{\infty}(\mathbb{R})=\left\{\sum_{d=1}^{m} u\left(t_{d}\right) \in\right.$ $C \mathbb{R}: \lim d=1 \mathrm{mtd} \rightarrow \infty d=1$ mutd $=0$,

(b) $\sup _{\mathbb{R}} \sum_{d=1}^{m} C_{\psi}\left(t_{d}\right)<\infty$;

(c) for an y $\sum_{d=1}^{m} f\left(t_{d}\right)=\sum_{d=1}^{m}(\boldsymbol{c} * \varphi)\left(t_{d}\right) \in$ $\sum_{d=1}^{m} V\left(\varphi\left(t_{d}\right)\right)$,

$\sum_{d=1}^{m} L[f]\left(t_{d}\right)=\sum_{d=1}^{m}(\boldsymbol{c} * \psi)\left(t_{d}\right) \quad$ converges absolutely and uniformly on $\mathbb{R}$.

Hence $\sum_{d=1}^{m} L[f]\left(t_{d}\right) \in C(\mathbb{R})$.

Proof .First assume $\sum_{d=1}^{m} l\left(t_{d}\right) \in L^{2}(\mathbb{R})$. Then $\sum_{d=1}^{m} \psi\left(t_{d}\right) \in C_{\infty}(\mathbb{R})$ by the Riemann-Lebesgue lemma since $\hat{\psi}(\xi)=\hat{\varphi}(\xi) \hat{l}(\xi) \in L^{1}(\mathbb{R})$. Since

$$
\begin{gathered}
\sum_{n \in \mathbb{Z}}|\hat{\psi}(\xi+2 n \pi)| \leq G_{\varphi}(\xi)^{\frac{1}{2}} G_{l}(\xi)^{\frac{1}{2}} \\
\|\left.\sum_{n \in \mathbb{Z}}|\widehat{\psi}(\xi+2 n \pi)|\right|_{L^{2[0,2 \pi]}} \leq \int_{0}^{2 \pi} G_{\varphi}(\xi) G_{l}(\xi) d \xi \\
\leq 2 \pi\left\|G_{\varphi}(\xi)\right\|_{L^{\infty}(\mathbb{R})}\|l\|_{L^{2}(\mathbb{R})}^{2} .
\end{gathered}
$$

Thus for any $\sum_{d=1}^{m} t_{d}$ in $\mathbb{R}$, we have by the Poisson summation formula (se [13])

$$
\begin{aligned}
\sum_{n \in \mathbb{Z}} \hat{\psi}(\xi+2 n \pi) & \prod_{d=1}^{m} e^{i t_{d}(\xi+2 n \pi)} \\
& =\sum_{n \in \mathbb{Z}} \sum_{d=1}^{m} \psi\left(t_{d}\right. \\
& +n) e^{-i n \xi} \text { in } L^{2}[0,2 \pi]
\end{aligned}
$$

Therefore for any $\sum_{d=1}^{m} t_{d}$ in $\mathbb{R}$

$$
\begin{gathered}
\sum_{d=1}^{m} C_{\psi}\left(t_{d}\right)=\sum_{n \in \mathbb{Z}} \sum_{d=1}^{m}\left|\psi\left(t_{d}+n\right)\right|^{2} \\
=\frac{1}{2 \pi} \| \sum_{n \in \mathbb{Z}} \sum_{d=1}^{m} \psi\left(t_{d}\right. \\
+n) e^{-i n \xi} \|_{L^{2}[0,2 \pi]}^{2} \\
=\frac{1}{2 \pi}\left\|\sum_{n \in \mathbb{Z}} \hat{\psi}(\xi+2 n \pi) \prod_{d=1}^{m} e^{i t_{d}(\xi+2 n \pi)}\right\|_{L^{2}[0,2 \pi]}^{2} \\
\leq\left\|G_{\varphi}(\xi)\right\|_{L^{\infty}(\mathbb{R})}\|l\|_{L^{2}(\mathbb{R})}
\end{gathered}
$$

By Young's inequality on the convolution product, $\|L[f]\|_{L^{\infty}(\mathbb{R})} \leq\|f\|_{L^{2}(\mathbb{R})}\|l\|_{L^{2}(\mathbb{R})}$ so that $L[\cdot]$ : $L^{2}(\mathbb{R}) \rightarrow L^{\infty}(\mathbb{R})$ is a bounded linear operator. Hence for any

$$
\begin{aligned}
\sum_{d=1}^{m} f\left(t_{d}\right)=\sum_{d=1}^{m}(c * \varphi)\left(t_{d}\right) & \\
& =\sum_{n \in \mathbb{Z}} \sum_{d=1}^{m} c(n) \varphi\left(t_{d}-n\right) \\
& \in \sum_{d=1}^{m} V\left(\varphi\left(t_{d}\right)\right) \\
\sum_{d=1}^{m} L[f]\left(t_{d}\right)= & \sum_{n \in \mathbb{Z}} \sum_{d=1}^{m} c(n) L\left[\varphi\left(t_{d}-n\right)\right] \\
& =\sum_{n \in \mathbb{Z}} \sum_{d=1}^{m} c(n) \psi\left(t_{d}-n\right)
\end{aligned}
$$


which converges absolutely and uniformly on R by (b). Now assume that $H_{\varphi}(\xi) \in L^{2}[0,2 \pi]$. The case $\hat{l}(\xi) \in L^{2}(\mathbb{R})$ is reduced to type (ii). So let $\hat{l}(\xi) \in L^{\infty}(\mathbb{R})$. Then $\hat{\varphi}(\xi) \in L^{2}(\mathbb{R}) \cap L^{1}(\mathbb{R})$ so that $\hat{\psi}(\xi)=\hat{\varphi}(\xi) \hat{l}(\xi) \in L^{2}(\mathbb{R}) \cap L^{1}(\mathbb{R})$ and so $\left.\psi(\xi) \in C_{\infty}(R) \cap L^{2}(\mathbb{R})\right)$. Since

$\sum_{n \in \mathbb{Z}}|\hat{\psi}(\xi+2 n \pi)| \leq\|l\|_{L^{\infty}(\mathbb{R})} H_{\varphi}(\xi)$,

we have again by the Poisson summation formula

$$
\begin{gathered}
\sum_{d=1}^{m} C_{\psi}\left(t_{d}\right)=\frac{1}{2 \pi} \| \sum_{n \in \mathbb{Z}} \hat{\psi}(\xi \\
+2 n \pi) \prod_{d=1}^{m} e^{i t_{d}(\xi+2 n \pi)} \|_{L^{2}[0,2 \pi]}^{2} \\
\leq\|l\|_{L^{\infty}(\mathbb{R})}^{2}\left\|H_{\varphi}(\xi)\right\|_{L^{2}[0,2 \pi]}^{2}
\end{gathered}
$$

so that $\sup _{\mathbb{R}} \sum_{d=1}^{m} C_{\psi}\left(t_{d}\right)<\infty$. For any $f \in L^{2}(\mathbb{R})$,

$$
\begin{gathered}
\sum_{d=1}^{m}\left\|L[f]\left(t_{d}\right)\right\|_{L^{2}(\mathbb{R})}=\|f * l\|_{L^{2}(\mathbb{R})} \\
=\frac{1}{\sqrt{2 \pi}}\|\hat{f}(\xi) \hat{l}(\xi)\|_{L^{2}(\mathbb{R})} \\
\leq\|\hat{l}\|_{L^{\infty}(\mathbb{R})}\|f\|_{L^{2}(\mathbb{R})} .
\end{gathered}
$$

Hence $L[\cdot]: L^{2}(\mathbb{R}) \rightarrow L^{2}(\mathbb{R})$ is a bounded linear operator so that for any

$$
\sum_{d=1}^{m} f\left(t_{d}\right)=\sum_{d=1}^{m}(\boldsymbol{c} * \varphi)\left(t_{d}\right) \in
$$

$\sum_{d=1}^{m} V\left(\varphi\left(t_{d}\right)\right), \sum_{d=1}^{m} L[f]\left(t_{d}\right)=\sum_{d=1}^{m}(\boldsymbol{c} *$

$\psi t d$ converges in $L 2(\mathbb{R})$. By (b), $d=1 m \boldsymbol{c} * \psi t d$ also converges absolutely and uniformly on $\mathbb{R}$.

By Lemma 3.1(b) , $\sum_{d=1}^{m} \psi\left(t_{d}\right) \in L^{2}(\mathbb{R})$ However, $\sum_{d=1}^{m}(\boldsymbol{c} * \psi)\left(t_{d}\right)$ may not converge in $L^{2}(\mathbb{R})$ unless $\left\{\sum_{d=1}^{m} \psi\left(t_{d}-n\right): n \in \mathbb{Z}\right\}$ is a Bessel sequence.

Lemma 3.1(b) improves Lemma 1 in [9], in which the proof uses $\sum_{d=1}^{m} l\left(t_{d}\right) \in L^{2}(\mathbb{R}) \cap L^{1}(\mathbb{R})$,

$\sup _{\mathbb{R}} \sum_{d=1}^{m} C_{\varphi}\left(t_{d}\right)<\infty$, and the integral version of Minkowski inequality. Note that the condition
$H_{\varphi}(\xi) \in L^{2}[0,2 \pi] \quad$ implies $\quad \sum_{d=1}^{m} \varphi\left(t_{d}\right) \in$ $L^{2}(\mathbb{R}) \cap C_{\infty}\left((\mathbb{R})\right.$ and $\sup _{\mathbb{R}} \sum_{d=1}^{m} C_{\varphi}\left(t_{d}\right)<\infty$. (see [13]). Note also that $H_{\varphi}(\xi) \in L^{2}[0,2 \pi]$ if $\hat{\varphi}(\xi)=O\left((1+|\xi|)^{-\left(1+\epsilon_{2}\right)}\right),\left(1+\epsilon_{2}\right)_{\left(1+\epsilon_{1}\right)}>$ $1, \epsilon_{1} \geq 0$, which holds e.g. for $\sum_{d=1}^{m} \varphi_{n}\left(t_{d}\right)=$ $\sum_{d=1}^{m}\left(\varphi_{0} * \varphi_{n-1}\right)\left(t_{d}\right)$ the cardinal B-spline of degree $n(\geq 1)$, where

$\varphi_{0}=\sum_{d=1}^{m} \chi_{[0,1)}\left(t_{d}\right)$. We have as a consequence of Lemma 3.1: Let $L[\cdot]$ be an LTI system with impulse response $\sum_{d=1}^{m} l\left(t_{d}\right)$ of type (i) or (ii) or (iii) as above and $\sum_{d=1}^{m} \psi\left(t_{d}\right)=\sum_{d=1}^{m} L[\varphi]\left(t_{d}\right)$. Then for any

$$
\sum_{d=1}^{m} f\left(t_{d}\right)=\sum_{d=1}^{m}(\mathcal{J} F)\left(t_{d}\right) \in \sum_{d=1}^{m} V\left(\varphi\left(t_{d}\right)\right), F(\xi)
$$$$
\in L^{2}[0,2 \pi]
$$

$$
\begin{aligned}
& \sum_{d=1}^{m} L[f]\left(t_{d}\right) \\
& =\sum_{d=1}^{m}\left\langle(\xi), \frac{1}{2 \pi} \overline{Z_{\psi}\left(t_{d}, \xi\right)}\right\rangle_{L^{2}[0,2 \pi]}
\end{aligned}
$$

since $L[\cdot]$ is a bounded linear operator from $L^{2}(\mathbb{R})$ into $L^{2}(\mathbb{R})$ or $L^{\infty}(\mathbb{R})$ and $\left\{\sum_{d=1}^{m} \psi\left(t_{d}-\right.\right.$ $n): n \in \mathbb{Z}\} \in[2, d=1 m t d \in \mathbb{R}$. Let $d=1 m \psi 1+\epsilon 1(t d)$ $=\sum_{d=1}^{m} L_{\left(1+\epsilon_{1}\right)}[\varphi]\left(t_{d}\right)$ and

$$
g_{\left(1+\epsilon_{1}\right)}(\xi)=\frac{1}{2 \pi} Z_{\psi_{\left(1+\epsilon_{1}\right)}}\left(\sigma_{\left(1+\epsilon_{1}\right)}, \xi\right), \epsilon_{1} \geq 0 .
$$

Then we have by (2)

$$
\begin{aligned}
& L_{\left(1+\epsilon_{1}\right)}[f]\left(\sigma_{\left(1+\epsilon_{1}\right)}+\left(1+\epsilon_{2}\right)_{\left(1+\epsilon_{1}\right)} n\right) \\
& =\left\langle F(\xi), \frac{1}{2 \pi} Z_{\psi_{\left(1+\epsilon_{1}\right)}}\left(\sigma_{\left(1+\epsilon_{1}\right)}\right.\right. \\
& =\left\langle F(\xi), \frac{\left.\left.+\left(1+\epsilon_{2}\right)_{\left(1+\epsilon_{1}\right)} n, \xi\right)\right\rangle_{L^{2}[0,2 \pi]}}{g_{\left(1+\epsilon_{1}\right)}(\xi)} e^{-i\left(1+\epsilon_{2}\right)_{\left(1+\epsilon_{1}\right)} n \xi}\right\rangle_{L^{2}[0,2 \pi]}
\end{aligned}
$$

for $\quad$ any $\quad \sum_{d=1}^{m} f\left(t_{d}\right)=\sum_{d=1}^{m}(\mathcal{J} F)\left(t_{d}\right) \in$ $\sum_{d=1}^{m} V\left(\varphi\left(t_{d}\right)\right)$ and $\epsilon_{1} \geq 0$. Then by (3) and the isomorphism $\mathcal{J}$ from $L^{2}[0,2 \pi]$ onto $\sum_{d=1}^{m} V\left(\varphi\left(t_{d}\right)\right)$, the sampling expansion (1) is equivalent to

$$
F(\xi)=\sum_{\epsilon_{1}=0}^{N} \sum_{n \in \mathbb{Z}}\left\langle F(\xi), \overline{g_{\left(1+\epsilon_{1}\right)}(\xi)} e^{-i\left(1+\epsilon_{2}\right)_{\left(1+\epsilon_{1}\right)} n \xi}\right\rangle_{L^{2}[0,2 \pi]} S_{\left(1+\epsilon_{1}\right), n}(\xi),
$$


$F(\xi) \in L^{2}[0,2 \pi], \quad$ where $\quad\left\{S_{\left(1+\epsilon_{1}\right), n}(\xi): \epsilon_{1} \geq\right.$ $0, n \in \mathbb{Z}\}$ is a series of frames or a Riesz basis of $L^{2}[0,2 \pi]$. This observation leads us to consider the problem when is $\left\{\overline{g_{\left(1+\epsilon_{1}\right)}(\xi)} e^{-i\left(1+\epsilon_{2}\right)_{\left(1+\epsilon_{1}\right)} n \xi}: \epsilon_{1} \geq\right.$ $0, n \in \mathbb{Z}\}$ a series of frames or a Riesz basis of $L^{2}[0,2 \pi]$. Note that

$$
\begin{gathered}
\left\{\overline{g_{\left(1+\epsilon_{1}\right)}(\xi)} e^{-i\left(1+\epsilon_{2}\right)_{\left(1+\epsilon_{1}\right)} n \xi}: \epsilon_{1} \geq 0, n \in \mathbb{Z}\right\}= \\
\left\{\overline{g_{\left(1+\epsilon_{1}\right), m_{\left(1+\epsilon_{1}\right)}}(\xi)} e^{-i\left(1+\epsilon_{2}\right) n \xi}: \epsilon_{1} \geq 0,1 \leq m_{\left(1+\epsilon_{1}\right)}\right. \\
\left.\leq \frac{\left(1+\epsilon_{2}\right)}{\left(1+\epsilon_{2}\right)_{\left(1+\epsilon_{1}\right)}}, n \in \mathbb{Z}\right\}
\end{gathered}
$$

where $\quad\left(1+\epsilon_{2}\right)=\operatorname{lcm}\left\{\left(1+\epsilon_{2}\right)_{\left(1+\epsilon_{1}\right)}: \epsilon_{1} \geq 0\right\}$ and

$g_{\left(1+\epsilon_{1}\right), m_{\left(1+\epsilon_{1}\right)}}(\xi)=$

$g_{\left(1+\epsilon_{1}\right)}(\xi) e^{i\left(1+\epsilon_{2}\right)_{\left(1+\epsilon_{1}\right)}\left(m_{\left(1+\epsilon_{1}\right)}-1\right) \xi}$ for $\epsilon_{1} \geq 0$. Let

$D$ be the unitary operator from $L^{2}[0,2 \pi]$ onto

$L^{2}(I)^{\left(1+\epsilon_{2}\right)}$, where $=\left[0, \frac{2 \pi}{\left(1+\epsilon_{2}\right)}\right]$, defined by

$$
D F=\left[F\left(\xi+(k-1) \frac{2 \pi}{\left(1+\epsilon_{2}\right)}\right)\right]_{k=1}^{\left(1+\epsilon_{2}\right)}, F(\xi) \in
$$

$L^{2}[0,2 \pi]$. We also let

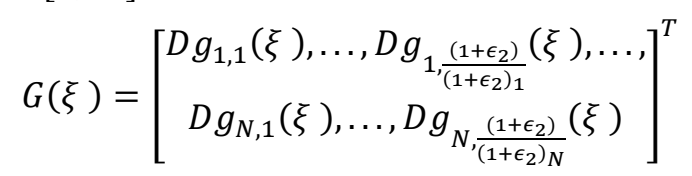

be a $\left(\sum_{\epsilon_{1}=0}^{N} \frac{\left(1+\epsilon_{2}\right)}{\left(1+\epsilon_{2}\right)_{\left(1+\epsilon_{1}\right)}}\right)$

$\times\left(1+\epsilon_{2}\right)$ matrix on $I$ and $\lambda_{m}(\xi), \lambda_{M}(\xi)$

be the smallest and the largest

eigenvalues of the positive semi-definite $\left(1+\epsilon_{2}\right) \times$ $\left(1+\epsilon_{2}\right)$ matrix $G(\xi) * G(\xi)$, respectively.

Lemma 3.2 . Let $\alpha_{G}=\left\|\lambda_{m}(\xi)\right\|_{0}$ and $\beta_{G}=\left\|\lambda_{M}(\xi)\right\|_{\infty}$ be the essential infimum of $\lambda_{m}(\xi)$ and the essential supremum of $\lambda_{M}(\xi)$ respectively.

Then

$\left\{\overline{g_{\left(1+\epsilon_{1}\right)}(\xi)} e^{-i\left(1+\epsilon_{2}\right)_{\left(1+\epsilon_{1}\right)} n \xi}: \epsilon_{1} \geq 0, n \in \mathbb{Z}\right\}$ is

(a) a Bessel sequence in $L^{2}[0,2 \pi]$ if and only if $\beta_{G}<\infty$ or equivalently

$\left\{Z_{\psi_{\left(1+\epsilon_{1}\right)}}\left(\sigma_{\left(1+\epsilon_{1}\right)}, \xi\right): \epsilon_{1} \geq 0\right\} \in L^{\infty}[0,2 \pi]$,

(b) a frame of $L^{2}[0,2 \pi]$ if and only if $0<\alpha_{G} \leq$ $\beta_{G}<\infty$, (c) a Riesz basis of $L^{2}[0,2 \pi]$ if and only if $0<\alpha_{G} \leq \beta_{G}<\infty$ and

$$
\sum_{\epsilon_{1}=0}^{N} \frac{\left(1+\epsilon_{2}\right)}{\left(1+\epsilon_{2}\right)_{\left(1+\epsilon_{1}\right)}}=1 .
$$

Proof. Since $\left\{\overline{g_{\left(1+\epsilon_{1}\right)}(\xi)} e^{-i\left(1+\epsilon_{2}\right)_{\left(1+\epsilon_{1}\right)} n \xi}: \epsilon_{1} \geq\right.$ $0, n \in \mathbb{Z}\}$ is a Bessel sequence or a series of frames or a Riesz basis of $L^{2}[0,2 \pi]$ if and only if

$$
\begin{gathered}
\left\{\overline{g_{\left(1+\epsilon_{1}\right), m_{\left(1+\epsilon_{1}\right)}}(\xi)} e^{-i\left(1+\epsilon_{2}\right) n \xi}: \epsilon_{1} \geq 0,1 \leq m_{\left(1+\epsilon_{1}\right)}\right. \\
\left.\leq \frac{\left(1+\epsilon_{2}\right)}{\left(1+\epsilon_{2}\right)_{\left(1+\epsilon_{1}\right)}}, n \in \mathbb{Z}\right\}
\end{gathered}
$$

is a Bessel sequence or a series of frames or a Riesz basis of $L^{2}[0,2 \pi]$ respectively, all of the conclusions follow from Lemma 3 in [9].

Note that in [9], the authors use the Fourier transform

$\hat{f}(\xi)=\int_{-\infty}^{\infty} \sum_{d=1}^{m} f\left(t_{d}\right) \prod_{d=1}^{m} e^{-2 \pi i t_{d} \xi} d t_{d} \quad$ so that they use $L^{2}[0,2 \pi]$ instead of $L^{2}[0,2 \pi]$.

Assume that $0<\alpha_{G} \leq \beta_{G}<\infty$ so that

$\left\{\overline{g_{\left(1+\epsilon_{1}\right)}(\xi)} e^{-\mathrm{i}\left(1+\epsilon_{2}\right)_{\left(1+\epsilon_{1}\right)} n \xi}: \epsilon_{1} \geq 0, n \in \mathbb{Z}\right\} \quad$ or equivalently

$$
\begin{gathered}
\left\{\overline{g_{\left(1+\epsilon_{1}\right), m_{\left(1+\epsilon_{1}\right)}}(\xi)} e^{-i\left(1+\epsilon_{2}\right) n \xi}: \epsilon_{1} \geq 0,1 \leq m_{\left(1+\epsilon_{1}\right)}\right. \\
\left.\leq \frac{\left(1+\epsilon_{2}\right)}{\left(1+\epsilon_{2}\right)_{\left(1+\epsilon_{1}\right)}}, n \in \mathbb{Z}\right\}
\end{gathered}
$$

is a series of frames of $L^{2}[0,2 \pi]$. Then we can show easily (see in [9]) that

$$
\begin{gathered}
\left\{\overline{g_{\left(1+\epsilon_{1}\right), m_{\left(1+\epsilon_{1}\right)}}(\xi)} e^{-i\left(1+\epsilon_{2}\right) n \xi}: \epsilon_{1} \geq 0,1 \leq m_{\left(1+\epsilon_{1}\right)}\right. \\
\left.\leq \frac{\left(1+\epsilon_{2}\right)}{\left(1+\epsilon_{2}\right)_{\left(1+\epsilon_{1}\right)}}, n \in \mathbb{Z}\right\}
\end{gathered}
$$

has a series of dual frames of the form

$$
\begin{aligned}
\left\{S_{\left(1+\epsilon_{1}\right), m_{\left(1+\epsilon_{1}\right)}}(\xi) e^{-i\left(1+\epsilon_{2}\right) n \xi}: \epsilon_{1} \geq 0,1\right. & \\
& \leq m_{\left(1+\epsilon_{1}\right)} \leq \frac{\left(1+\epsilon_{2}\right)}{\left(1+\epsilon_{2}\right)_{\left(1+\epsilon_{1}\right)}}, n \\
& \in \mathbb{Z}\}
\end{aligned}
$$

with 
$S_{\left(1+\epsilon_{1}\right), m_{\left(1+\epsilon_{1}\right)}}(\xi) \in L^{\infty}[0,2 \pi]$ for $\epsilon_{1} \geq 0$ and

$1 \leq m_{\left(1+\epsilon_{1}\right)} \leq \frac{\left(1+\epsilon_{2}\right)}{\left(1+\epsilon_{2}\right)_{\left(1+\epsilon_{1}\right)}}$ satisfying

$$
\begin{aligned}
& {\left[\begin{array}{c}
D S_{1,1}(\xi), \ldots, D S_{1, \frac{\left(1+\epsilon_{2}\right)}{\left(1+\epsilon_{2}\right)_{1}}}(\xi), \ldots, \\
D S_{N, 1}(\xi), \ldots, D S_{N, \frac{\left(1+\epsilon_{2}\right)}{\left(1+\epsilon_{2}\right)_{N}}}(\xi)
\end{array}\right]} \\
& =\frac{\left(1+\epsilon_{2}\right)}{2 \pi}\left[G(\xi)^{\dagger}\right. \\
& \left.+B(\xi)\left(I-G(\xi) G(\xi)^{\dagger}\right)\right]
\end{aligned}
$$

where

$$
G(\xi)^{\dagger}=\left[G(\xi)^{*} G(\xi)\right]^{-1} G(\xi)^{*}
$$

is the pseudo-inverse of $G(\xi), B(\xi)$ is any

$$
\left(1+\epsilon_{2}\right) \times \sum_{\epsilon_{1}=0}^{N} \frac{\left(1+\epsilon_{2}\right)}{\left(1+\epsilon_{2}\right)_{\left(1+\epsilon_{1}\right)}}
$$

matrix with entries in $L^{\infty}(I)$, and $I$ is the

$$
\begin{aligned}
& \left(\sum_{\epsilon_{1}=0}^{N} \frac{\left(1+\epsilon_{2}\right)}{\left(1+\epsilon_{2}\right)_{\left(1+\epsilon_{1}\right)}}\right) \\
& \times\left(\sum_{\epsilon_{1}=0}^{N} \frac{\left(1+\epsilon_{2}\right)}{\left(1+\epsilon_{2}\right)_{\left(1+\epsilon_{1}\right)}}\right) \text { identity matrix. }
\end{aligned}
$$

In particular, when we choose $B(\xi)=0$ in (5), we have the canonical a series of dual frames of the frames

$$
\begin{gathered}
\left\{\overline{g_{\left(1+\epsilon_{1}\right), m_{\left(1+\epsilon_{1}\right)}}(\xi)} e^{-i\left(1+\epsilon_{2}\right) n \xi}: \epsilon_{1} \geq 0,1 \leq m_{\left(1+\epsilon_{1}\right)}\right. \\
\left.\leq \frac{\left(1+\epsilon_{2}\right)}{\left(1+\epsilon_{2}\right)_{\left(1+\epsilon_{1}\right)}}, n \in \mathbb{Z}\right\} .
\end{gathered}
$$

We are now ready to give the foolowing results (see [22]). We first discuss the sampling expansion (1), which is a series of frames expansion in $\sum_{d=1}^{m} V\left(\varphi\left(t_{d}\right)\right)$.

Theorem 3.3. Let $\alpha_{G}$ and $\beta_{G}$ be the same as in Lemma 3.2. Assume $\beta_{G}<\infty$. Then the following are all equivalent.

(a) There is a series of frames

$$
\begin{aligned}
\left\{\sum_{d=1}^{m} S_{\left(1+\epsilon_{1}\right), m_{\left(1+\epsilon_{1}\right)}}\left(t_{d}-\left(1+\epsilon_{2}\right) n\right): \epsilon_{1} \geq 0,1\right. \\
\left.\leq m_{\left(1+\epsilon_{1}\right)} \leq \frac{\left(1+\epsilon_{2}\right)}{\left(1+\epsilon_{2}\right)_{\left(1+\epsilon_{1}\right)}}, n \in \mathbb{Z}\right\}
\end{aligned}
$$

of $\sum_{d=1}^{m} V\left(\varphi\left(t_{d}\right)\right)$ for which

$$
\sum_{d=1}^{m} f\left(t_{d}\right)
$$

$$
\begin{aligned}
& =\sum_{\epsilon_{1}=0}^{N} \sum_{m_{\left(1+\epsilon_{1}\right)}=1}^{\frac{c_{\left.1+\epsilon_{2}\right)}}{\left(1+\epsilon_{2}\right)_{\left(1+\epsilon_{1}\right)}}} \sum_{n \in \mathbb{Z}} \sum_{d=1}^{m} L_{\left(1+\epsilon_{1}\right)}[f]\left(\sigma_{\left(1+\epsilon_{1}\right)}\right. \\
& +\left(1+\epsilon_{2}\right)_{\left(1+\epsilon_{1}\right)}\left(m_{\left(1+\epsilon_{1}\right)}-1\right) \\
& \left.+\left(1+\epsilon_{2}\right) n\right) s_{d\left(1+\epsilon_{1}\right), m_{\left(1+\epsilon_{1}\right)}}\left(t_{d}-\left(1+\epsilon_{2}\right) n\right)
\end{aligned}
$$

$$
\sum_{d=1}^{m} f\left(t_{d}\right) \in \sum_{d=1}^{m} V\left(\varphi\left(t_{d}\right)\right)
$$

(b) There is a series of frames

$$
\left.\sum_{d=1}^{m} s_{\left(1+\epsilon_{1}\right), n}\left(t_{d}\right): \epsilon_{1} \geq 0, n \in \mathbb{Z}\right\}
$$

of $\sum_{d=1}^{m} V\left(\varphi\left(t_{d}\right)\right)$ for which

$$
\begin{aligned}
\sum_{d=1}^{m} f\left(t_{d}\right)=\sum_{\epsilon_{1}=0}^{N} & \sum_{n \in \mathbb{Z}} \sum_{d=1}^{m} L_{\left(1+\epsilon_{1}\right)}[f]\left(\sigma_{\left(1+\epsilon_{1}\right)}\right. \\
& \left.+\left(1+\epsilon_{2}\right)_{\left(1+\epsilon_{1}\right)} n\right) s_{d\left(1+\epsilon_{1}\right), n}\left(t_{d}\right)
\end{aligned}
$$

$$
\sum_{d=1}^{m} f\left(t_{d}\right) \in \sum_{d=1}^{m} V\left(\varphi\left(t_{d}\right)\right)
$$

(c) $0<\alpha_{G}$.

Proof. Assume $\beta_{G}<\infty$. Then by Lemma 3.2 $\left\{\overline{g_{\left(1+\epsilon_{1}\right)}(\xi)} e^{-i\left(1+\epsilon_{2}\right)_{\left(1+\epsilon_{1}\right)} n \xi}: \epsilon_{1} \geq 0, n \in \mathbb{Z}\right\}$ is a Bessel sequence in $L^{2}[0,2 \pi]$. First (a) implies (b) trivially. Assume (b). Applying the isomorphism $\mathcal{J}^{-1}$ to (7) gives by (3)

$$
F(\xi)=\sum_{m_{\left(1+\epsilon_{1}\right)}=1}^{\frac{\left(1+\epsilon_{2}\right)}{\left(1+\epsilon_{2}\right)\left(1+\epsilon_{1}\right)}} \sum_{n \in \mathbb{Z}}\left\langle F(\xi), \overline{g_{J}(\xi)} e^{-i\left(1+\epsilon_{2}\right)_{\left(1+\epsilon_{1}\right)} n \xi}\right\rangle_{L^{2}[0,2 \pi]} s_{d\left(1+\epsilon_{1}\right), n}(\xi),
$$




$$
F(\xi) \in L^{2}[0,2 \pi]
$$

where $\left\{\sum_{d=1}^{m} s_{d\left(1+\epsilon_{1}\right), n}\left(t_{d}\right): 0 \leq \epsilon_{1} \leq N, n \in \mathbb{Z}\right\}$ is a series of frames of $L^{2}[0,2 \pi]$. Then the Bessel sequence $\quad\left\{\overline{g_{\left(1+\epsilon_{1}\right)}(\xi)} e^{-i\left(1+\epsilon_{2}\right)_{\left(1+\epsilon_{1}\right)}^{n \xi}}: 0 \leq \epsilon_{1} \leq\right.$ $N, n \in \mathbb{Z}\}$ is in fact a series of dual frames of $\left\{\sum_{d=1}^{m} s_{d\left(1+\epsilon_{1}\right), n}\left(t_{d}\right): 0 \leq \epsilon_{1} \leq N, n \in \mathbb{Z}\right\}$ (see [2]). Hence (c) must hold by Lemma 3.2. Finally assume (c). Then $0<\alpha_{G} \leq \beta_{G}<\infty$ that

$$
\left\{\overline{g_{\left(1+\epsilon_{1}\right), m_{\left(1+\epsilon_{1}\right)}}(\xi)} e^{-i\left(1+\epsilon_{2}\right) n \xi}: \epsilon_{1} \geq 0,1 \leq\right.
$$

$\left.m_{\left(1+\epsilon_{1}\right)} \leq \frac{\left(1+\epsilon_{2}\right)}{\left(1+\epsilon_{2}\right)_{\left(1+\epsilon_{1}\right)}}, n \in \mathbb{Z}\right\}$ is a series of frames of $L^{2}[0,2 \pi]$. Then we have a frame expansion on $L^{2}[0,2 \pi]$

$$
F(\xi)=\sum_{\epsilon_{1}=0}^{N} \sum_{m_{\left(1+\epsilon_{1}\right)}=1}^{\frac{\left(1+\epsilon_{2}\right)}{\left(1+\epsilon_{2}\right)\left(1+\epsilon_{1}\right)}} \sum_{n \in \mathbb{Z}}\left(\left\langle F(\xi), \overline{g_{\left(1+\epsilon_{1}\right), m_{\left(1+\epsilon_{1}\right)}}(\xi)} e^{-i\left(1+\epsilon_{2}\right) n \xi}\right\rangle_{L^{2}[0,2 \pi]} S_{\left(1+\epsilon_{1}\right), m_{\left(1+\epsilon_{1}\right)}}\right.
$$

$$
\left.(\xi) e^{-i\left(1+\epsilon_{2}\right) n \xi}\right), F(\xi) \in L^{2}[0,2 \pi]
$$

where $S_{\left(1+\epsilon_{1}\right), m_{\left(1+\epsilon_{1}\right)}}(\xi)$ 's are given by (5). Then the sampling expansion (6) comes from (8) by applying the isomorphism $\mathcal{J}$ since

$$
\begin{aligned}
& \left\langle F(\xi), g_{\left(1+\epsilon_{1}\right), m_{\left(1+\epsilon_{1}\right)}}(\xi) e^{-i\left(1+\epsilon_{2}\right) n \xi}\right\rangle_{L^{2}[0,2 \pi]} \\
& =\left\langle F(\xi), \frac{1}{2 \pi} \overline{Z_{\psi J}\left(\sigma_{\left(1+\epsilon_{1}\right)}+\left(1+\epsilon_{2}\right)_{\left(1+\epsilon_{1}\right)}\left(m_{\left(1+\epsilon_{1}\right)}-1\right)+\left(1+\epsilon_{2}\right) n, \xi\right)}\right\rangle_{L^{2}[0,2 \pi]} \\
& =L_{\left(1+\epsilon_{1}\right)}[f]\left(\sigma_{\left(1+\epsilon_{1}\right)}+\left(1+\epsilon_{2}\right)_{\left(1+\epsilon_{1}\right)}\left(m_{\left(1+\epsilon_{1}\right)}-1\right)+\left(1+\epsilon_{2}\right) n\right) \\
& \text { for } \sum_{d=1}^{m}(\mathcal{J} F)\left(t_{d}\right)=\sum_{d=1}^{m} f\left(t_{d}\right)
\end{aligned}
$$

Note that when $0<\alpha_{G} \leq \beta_{G}<\infty$, the sampling series (6) converges not only in $L^{2}(\mathbb{R})$ but also uniformly on any subset of $\mathbb{R}$, on which $\sum_{d=1}^{m} C_{\varphi}\left(t_{d}\right)$ is bounded. Moreover since $\alpha_{G}>0$, the rank of

$$
\begin{gathered}
G(\xi) \text { is }\left(1+\epsilon_{2}\right) \text { a. e. so that } 1 \\
\leq \sum_{\epsilon_{1}=0}^{N} \frac{1}{\left(1+\epsilon_{2}\right)_{\left(1+\epsilon_{1}\right)}}
\end{gathered}
$$

which means that the total sampling rate

$$
\sum_{\epsilon_{1}=0}^{N} \frac{1}{\left(1+\epsilon_{2}\right)_{\left(1+\epsilon_{1}\right)}}
$$

of the sampling expansion (6)must be at least 1 , the Nyquist sampling rate for

signals in $\sum_{d=1}^{m} V\left(\varphi\left(t_{d}\right)\right)$. In the extreme case we have:

Theorem 3.4.Let $\alpha_{G}$ and $\beta_{G}$ be the same as in Lemma 3.2. Then there is a series of Riesz bases

$$
\left\{\sum_{d=1}^{m} S_{\left(1+\epsilon_{1}\right), m_{\left(1+\epsilon_{1}\right), n}}\left(t_{d}\right): \epsilon_{1} \geq 0,1 \leq\right.
$$

$\left.m_{\left(1+\epsilon_{1}\right)} \leq \frac{\left(1+\epsilon_{2}\right)}{\left(1+\epsilon_{2}\right)_{\left(1+\epsilon_{1}\right)}}, n \in \mathbb{Z}\right\} \quad$ of $\quad \sum_{d=1}^{m} V\left(\varphi\left(t_{d}\right)\right)$ for which

$$
\begin{aligned}
& \sum_{d=1}^{m} f\left(t_{d}\right) \\
& =\sum_{\epsilon_{1}=0}^{N} \sum_{m_{\left(1+\epsilon_{1}\right)}=1}^{\frac{\left(1+\epsilon_{2}\right)}{\left(1+\epsilon_{2}\right)_{\left(1+\epsilon_{1}\right)}}} \sum_{n \in \mathbb{Z}} \sum_{d=1}^{m}\left(L _ { ( 1 + \epsilon _ { 1 } ) } [ f ] \left(\sigma_{\left(1+\epsilon_{1}\right)}\right.\right. \\
& +\left(1+\epsilon_{2}\right)_{\left(1+\epsilon_{1}\right)}\left(m_{\left(1+\epsilon_{1}\right)}-1\right) \\
& \left.+\left(1+\epsilon_{2}\right) n\right) S_{\left.\left(1+\epsilon_{1}\right), m_{\left(1+\epsilon_{1}\right), n}\left(t_{d}\right)\right)} \\
& \quad \sum_{d=1}^{m} f\left(t_{d}\right) \in \sum_{d=1}^{m} V\left(\varphi\left(t_{d}\right)\right)
\end{aligned}
$$

if and only if $0<\alpha_{G} \leq \beta_{G}<$ $\infty$ and $\sum_{\epsilon_{1}=0}^{N} \frac{\left(1+\epsilon_{2}\right)}{\left(1+\epsilon_{2}\right)_{\left(1+\epsilon_{1}\right)}}=1$. In this case, we also have 
(i) $\sum_{d=1}^{m} S_{\left(1+\epsilon_{1}\right), m_{\left(1+\epsilon_{1}\right)}}\left(t_{d}-\left(1+\epsilon_{2}\right) n\right): \epsilon_{1} \geq 0$,

$1 \leq m_{\left(1+\epsilon_{1}\right)} \leq \frac{\left(1+\epsilon_{2}\right)}{\left(1+\epsilon_{2}\right)_{\left(1+\epsilon_{1}\right)}}$, and $n \in \mathbb{Z}$

(ii)

$$
\begin{aligned}
L_{\left(1+\epsilon_{1}\right)}\left[s_{k, m_{k}}\right] & \sigma_{\left(1+\epsilon_{1}\right)} \\
& +\left(1+\epsilon_{2}\right)_{\left(1+\epsilon_{1}\right)}\left(m_{\left(1+\epsilon_{1}\right)}-1\right) \\
& \left.+\left(1+\epsilon_{2}\right) n\right)=\delta_{\left(1+\epsilon_{1}\right), k} \delta_{n, 0}
\end{aligned}
$$

for $0 \leq \epsilon_{1}, k \leq N$ and $n \in \mathbb{Z}$.

$$
\sum_{\epsilon_{1}=0}^{N} \frac{\left(1+\epsilon_{2}\right)}{\left(1+\epsilon_{2}\right)_{\left(1+\epsilon_{1}\right)}}=1
$$

Then by Lemma 3.2,

$$
\begin{gathered}
\left\{\overline{g_{\left(1+\epsilon_{1}\right), m_{\left(1+\epsilon_{1}\right)}}(\xi)} e^{-i\left(1+\epsilon_{2}\right) n \xi}: \epsilon_{1} \geq 0,1 \leq m_{\left(1+\epsilon_{1}\right)}\right. \\
\left.\leq \frac{\left(1+\epsilon_{2}\right)}{\left(1+\epsilon_{2}\right)_{\left(1+\epsilon_{1}\right)}}, n \in \mathbb{Z}\right\}
\end{gathered}
$$

Proof .Assume $0<\alpha_{G} \leq \beta_{G}<\infty$ and is a series of Riesz bases of $L^{2}[0,2 \pi]$. Then we have

$$
\begin{gathered}
F(\xi)=\sum_{\epsilon_{1}=0}^{N} \sum_{m_{\left(1+\epsilon_{1}\right)}}^{\frac{\left(1+\epsilon_{2}\right)}{\left(1+\epsilon_{2}\right)\left(1+\epsilon_{1}\right)}} \sum_{n \in \mathbb{Z}}\left\langle F(\xi), \frac{g_{\left(1+\epsilon_{1}\right), m_{\left(1+\epsilon_{1}\right)}}(\xi)}{\left.g^{-i\left(1+\epsilon_{2}\right) n \xi}\right\rangle_{L^{2}[0,2 \pi]} S_{\left(1+\epsilon_{1}\right), m_{\left(1+\epsilon_{1}\right)}}(\xi) e^{-i\left(1+\epsilon_{2}\right) n \xi}}\right. \\
, F(\xi) \in L^{2}[0,2 \pi],
\end{gathered}
$$

where

$$
\begin{aligned}
& \left\{S_{\left(1+\epsilon_{1}\right), m_{\left(1+\epsilon_{1}\right)}}(\xi) e^{-i\left(1+\epsilon_{2}\right) n \xi}: \epsilon_{1} \geq 0,1 \leq m_{\left(1+\epsilon_{1}\right)}\right. \\
& \left.\qquad \leq \frac{\left(1+\epsilon_{2}\right)}{\left(1+\epsilon_{2}\right)_{\left(1+\epsilon_{1}\right)}}, n \in \mathbb{Z}\right\} \\
& \text { is the dual of } \\
& \left\{\frac{g_{\left(1+\epsilon_{1}\right), m_{\left(1+\epsilon_{1}\right)}(\xi)} e^{-i\left(1+\epsilon_{2}\right) n \xi}: \epsilon_{1} \geq 0,1 \leq m_{\left(1+\epsilon_{1}\right)}}{\leq}\right. \\
& \left.\leq \frac{\left(1+\epsilon_{2}\right)}{\left(1+\epsilon_{2}\right)_{\left(1+\epsilon_{1}\right)}}, n \in \mathbb{Z}\right\}
\end{aligned}
$$$$
\sum_{d=1}^{m} S_{\left(1+\epsilon_{1}\right), m_{\left(1+\epsilon_{1}\right), n}}\left(t_{d}\right)=\mathcal{J}\left((\xi) e^{-i\left(1+\epsilon_{2}\right) n \xi}\right)
$$

$$
=\sum_{d=1}^{m} S_{\left(1+\epsilon_{1}\right), m_{\left(1+\epsilon_{1}\right)}}\left(t_{d}-\left(1+\epsilon_{2}\right) n\right)
$$

and

$\mathcal{J}\left(S_{\left(1+\epsilon_{1}\right), m_{\left(1+\epsilon_{1}\right)}}(\xi)\right)=$

$\sum_{d=1}^{m} S_{\left(1+\epsilon_{1}\right), m_{\left(1+\epsilon_{1}\right)}}\left(t_{d}\right)$. Conversely assume that the series of Riesz bases expansions (9) holds on Applying the isomorphism $\mathcal{J}$ to (10) gives (9), where $\sum_{d=1}^{m} V\left(\varphi\left(t_{d}\right)\right)$. Applying the isomorphism $\mathcal{J}^{-1}$ to (9) gives

$$
\begin{gathered}
F(\xi)=\sum_{\epsilon_{1}=0}^{N} \sum_{m_{\left(1+\epsilon_{1}\right)}}^{\frac{\left(1+\epsilon_{2}\right)}{\left(1+\epsilon_{2}\right)}\left(1+\epsilon_{1}\right)} \sum_{n \in \mathbb{Z}}\left(\left\langle F(\xi), \frac{g_{\left(1+\epsilon_{1}\right), m_{\left(1+\epsilon_{1}\right)}}(\xi)}{-i\left(1+\epsilon_{2}\right) n \xi}\right\rangle_{L^{2}[0,2 \pi]} \mathcal{J}^{-1}\left(S_{\left.\left(1+\epsilon_{1}\right), m_{\left(1+\epsilon_{1}\right)}\right)}\right)(\xi)\right) \\
, F(\xi) \in L^{2}[0,2 \pi]
\end{gathered}
$$

which is a series of Riesz bases expansions on $L^{2}[0,2 \pi]$. Then

$$
\begin{gathered}
\left\{\overline{g_{\left(1+\epsilon_{1}\right), m_{\left(1+\epsilon_{1}\right)}}(\xi)} e^{-i\left(1+\epsilon_{2}\right) n \xi}: \epsilon_{1} \geq 0,1 \leq m_{\left(1+\epsilon_{1}\right)}\right. \\
\left.\leq \frac{\left(1+\epsilon_{2}\right)}{\left(1+\epsilon_{2}\right)_{\left(1+\epsilon_{1}\right)}}, n \in \mathbb{Z}\right\}
\end{gathered}
$$

must be a series of Riesz bases of $L^{2}[0,2 \pi]$ so that $0<\alpha_{G} \leq \beta_{G}<\infty$ and $\sum_{\epsilon_{1}=0}^{N} \frac{\left(1+\epsilon_{2}\right)}{\left(1+\epsilon_{2}\right)_{\left(1+\epsilon_{1}\right)}}=1$ by Lemma 3.2. As the a series of the dual Riesz bases of

$$
\begin{gathered}
\left\{\overline{g_{\left(1+\epsilon_{1}\right), m_{\left(1+\epsilon_{1}\right)}}(\xi)} e^{-i\left(1+\epsilon_{2}\right) n \xi}: \epsilon_{1} \geq 0,1 \leq m_{\left(1+\epsilon_{1}\right)}\right. \\
\left.\leq \frac{\left(1+\epsilon_{2}\right)}{\left(1+\epsilon_{2}\right)_{\left(1+\epsilon_{1}\right)}}, n \in \mathbb{Z}\right\}, \\
\left\{\sum_{d=1}^{m} \mathcal{J}^{-1}\left(S_{\left.\left(1+\epsilon_{1}\right), m_{\left(1+\epsilon_{1}\right)}\right)}\right)\left(t_{d}\right): \epsilon_{1} \geq 0,1 \leq m_{\left(1+\epsilon_{1}\right)}\right. \\
\left.\leq \frac{\left(1+\epsilon_{2}\right)}{\left(1+\epsilon_{2}\right)_{\left(1+\epsilon_{1}\right)}}, n \in \mathbb{Z}\right\}
\end{gathered}
$$

must be of the form 


$$
\begin{aligned}
\left\{S_{\left(1+\epsilon_{1}\right), m_{\left(1+\epsilon_{1}\right)}}(\xi) e^{-i\left(1+\epsilon_{2}\right) n \xi}: \epsilon_{1} \geq 0,1\right. & \\
& \leq m_{\left(1+\epsilon_{1}\right)} \leq \frac{\left(1+\epsilon_{2}\right)}{\left(1+\epsilon_{2}\right)_{\left(1+\epsilon_{1}\right)}}, n \\
& \in \mathbb{Z}\}
\end{aligned}
$$

where

$$
\begin{gathered}
\left\{S_{\left(1+\epsilon_{1}\right), m_{\left(1+\epsilon_{1}\right)}}(\xi): \epsilon_{1} \geq 0,1 \leq m_{\left(1+\epsilon_{1}\right)}\right. \\
\left.\leq \frac{\left(1+\epsilon_{2}\right)}{\left(1+\epsilon_{2}\right)_{\left(1+\epsilon_{1}\right)}}\right\}
\end{gathered}
$$

satisfy (5) with $B(\xi)=0$. Hence

$$
\begin{aligned}
& \sum_{d=1}^{m} S_{\left(1+\epsilon_{1}\right), m_{\left(1+\epsilon_{1}\right)}, n}\left(t_{d}\right) \\
& =\mathcal{J}\left(S_{\left(1+\epsilon_{1}\right), m_{\left(1+\epsilon_{1}\right)}}(\xi) e^{-i\left(1+\epsilon_{2}\right) n \xi}\right) \\
& =\sum_{d=1}^{m} S_{\left(1+\epsilon_{1}\right), m_{\left(1+\epsilon_{1}\right)}}\left(t_{d}-\left(1+\epsilon_{2}\right) n\right), \epsilon_{1} \geq 0, n \\
& \in \mathbb{Z} .
\end{aligned}
$$

Finally, we have

$$
\sum_{d=1}^{m} s_{k, m k}\left(t_{d}\right)=
$$

$$
\begin{aligned}
\sum_{\epsilon_{1}=0}^{N} \sum_{m_{\left(1+\epsilon_{1}\right)}}^{\frac{\left(1+\epsilon_{2}\right)}{\left(1+\epsilon_{2}\right)} \sum_{\left(1+\epsilon_{1}\right)}} \sum_{n \in \mathbb{Z}} \sum_{d=1}^{m}\left(L _ { ( 1 + \epsilon _ { 1 } ) } [ s _ { k , m _ { k } } ] \left(\sigma_{\left(1+\epsilon_{1}\right)}\right.\right. \\
+\left(1+\epsilon_{2}\right)_{\left(1+\epsilon_{1}\right)}\left(m_{\left(1+\epsilon_{1}\right)}-1\right) \\
\left.+\left(1+\epsilon_{2}\right) n\right) S_{\left(1+\epsilon_{1}\right), m_{\left(1+\epsilon_{1}\right)}}\left(t_{d}\right. \\
\\
\left.\left.-\left(1+\epsilon_{2}\right) n\right)\right)
\end{aligned}
$$

So that

$L_{\left(1+\epsilon_{1}\right)}\left[s_{k, m_{k}}\right]\left(\sigma_{\left(1+\epsilon_{1}\right)}+\left(1+\epsilon_{2}\right)_{\left(1+\epsilon_{1}\right)}\left(m_{\left(1+\epsilon_{1}\right)}-\right.\right.$ $1+1+\epsilon 2 n=\delta 1+\epsilon 11, k \delta n, 0$.

When $N=1$, write $L_{1}[\cdot], \sum_{d=1}^{m} l_{1}\left(t_{d}\right), \sigma_{1},(1+$ $\epsilon 21$, and $d=1 m \psi 1(t d)$ as $L[\cdot]$,

$$
\sum_{d=1}^{m} l\left(t_{d}\right), \sigma,\left(1+\epsilon_{2}\right) \text {, and } \sum_{d=1}^{m} \psi\left(t_{d}\right)
$$

Corollary 3.5. (Cf. Theorem 3.1 in [11].) Let $N=1$. Then there is a series of Riesz bases $\left\{\sum_{d=1}^{m} s_{n}\left(t_{d}\right): n \in \mathbb{Z}\right\}$ of $\sum_{d=1}^{m} V\left(\varphi\left(t_{d}\right)\right)$ such that

$$
\begin{aligned}
\sum_{d=1}^{m} f\left(t_{d}\right)=\sum_{n \in \mathbb{Z}} \sum_{d=1}^{m} L[f](\sigma & \\
& \left.+\left(1+\epsilon_{2}\right) n\right) s_{n}\left(t_{d}\right), \sum_{d=1}^{m} f\left(t_{d}\right)
\end{aligned}
$$

$$
\in \sum_{d=1}^{m} V\left(\varphi\left(t_{d}\right)\right)
$$

if and only if $\epsilon_{2}=0$ and

$$
0<\left\|Z_{\psi}(\sigma, \xi)\right\|_{0} \leq\left\|Z_{\psi}(\sigma, \xi)\right\|_{\infty} .
$$

In this case, we also have

(i) $\sum_{d=1}^{m} s_{n}\left(t_{d}\right)=\sum_{d=1}^{m} s\left(t_{d}-n\right), n \in \mathbb{Z}$,

(ii) $\hat{s}(\xi)=\frac{\widehat{\varphi}(\xi)}{z_{\psi}(\sigma, \xi)}$,

(iii) $L[s](\sigma+n)=\delta_{n, 0}, n \in \mathbb{Z} .(13)$

Proof .Note that for $\epsilon_{2}=0, G(\xi)=\frac{1}{2 \pi} Z_{\psi}(\sigma, \xi)$ and $\lambda_{m}(\xi)=\lambda_{M}(\xi)=\left(\frac{1}{2 \pi}\right)^{2}\left|Z_{\psi}(\sigma, \xi)\right|^{2}$ so that $0<\alpha_{G} \leq \beta_{G}<\infty$ if and only if (12) holds. Therefore, everything except (13) follows from Theorem 3.4. Finally applying (11) to $\sum_{d=1}^{m} \varphi\left(t_{d}\right)$ gives

$$
\sum_{d=1}^{m} \varphi\left(t_{d}\right)=\sum_{n \in \mathbb{Z}} \sum_{d=1}^{m} \psi(\sigma+n) s\left(t_{d}-n\right)
$$

from which we have (13) by taking the Fourier transform. When $\sum_{d=1}^{m} l\left(t_{d}\right)=\sum_{d=1}^{m} \delta\left(t_{d}\right)$ so that $L[\cdot]$ is the identity operator, Corollary 3.5 reduces to a series of regular shifted sampling on $\sum_{d=1}^{m} V\left(\varphi\left(t_{d}\right)\right)$ (see Theorem 3.3 in [13]).

Remark 3.6 .In (1), we may allow rational sampling periods. If $\left(1+\epsilon_{2}\right)_{\left(1+\epsilon_{1}\right)}=\frac{p_{\left(1+\epsilon_{1}\right)}}{q_{\left(1+\epsilon_{1}\right)}}$, where $p_{\left(1+\epsilon_{1}\right)}$ and $q_{\left(1+\epsilon_{1}\right)}$ are coprime positive integers, then

$$
\left[L_{\left(1+\epsilon_{1}\right)}[f]\left(\sigma_{\left(1+\epsilon_{1}\right)}+\left(1+\epsilon_{2}\right)_{\left(1+\epsilon_{1}\right)} n\right): n \in \mathbb{Z}\right]
$$
$=$

$\left[L_{\left(1+\epsilon_{1}\right)}[f]\left(\sigma_{\left(1+\epsilon_{1}\right)}+\left(1+\epsilon_{2}\right)_{\left(1+\epsilon_{1}\right)}(k-1)+\right.\right.$ $p 1+\epsilon 1 n: 1 \leq k \leq q 1+\epsilon 1, n \in \mathbb{Z}$. 
Hence the case of rational sampling periods $\left\{\left(1+\epsilon_{2}\right)_{\left(1+\epsilon_{1}\right)}\right\}_{\epsilon_{1}=0}^{N}$ can be reduced to the case of integer sampling periods $\left\{p_{\left(1+\epsilon_{1}\right)}\right\}_{\epsilon_{1}=0}^{N}=1$ by extending the number of LTI systems involved. For example when $N=1$, we have:

Corollary 3.7. Let $N=1$ and $q \geq 2$ be an integer. Assume $Z_{\psi}\left(\sigma_{\left(1+\epsilon_{1}\right)}, \xi\right) \in L^{\infty}[0,2 \pi]$, $0 \leq \epsilon_{1} \leq q-1$,

where $\sigma_{\left(1+\epsilon_{1}\right)}=\sigma+\frac{1}{q-1}\left(\epsilon_{1}\right)$. Then the following are all equivalent.

(a) There is a series of frames $\left\{\sum_{d=1}^{m} s_{n}\left(t_{d}\right): n \in\right.$ $\mathbb{Z}\}$ of $\sum_{d=1}^{m} V\left(\varphi\left(t_{d}\right)\right)$ for which

$$
\begin{aligned}
\sum_{d=1}^{m} f\left(t_{d}\right)=\sum_{n \in \mathbb{Z}} \sum_{d=1}^{m} L[f](\sigma & \\
& \left.+\frac{1}{q-1} n\right) s_{n}\left(t_{d}\right), \sum_{d=1}^{m} f\left(t_{d}\right) \\
& \in \sum_{d=1}^{m} V\left(\varphi\left(t_{d}\right)\right) .
\end{aligned}
$$

(b) There is a series of frames $\left\{\sum_{d=1}^{m} s_{\left(1+\epsilon_{1}\right)}\left(t_{d}-n\right): 0 \leq \epsilon_{1} \leq q-1, n \in \mathbb{Z}\right\} \quad$ of $\sum_{d=1}^{m} V\left(\varphi\left(t_{d}\right)\right)$ for which

$$
\begin{gathered}
\sum_{d=1}^{m} f\left(t_{d}\right)=\sum_{d=1}^{m} \sum_{\epsilon_{1}=0}^{q-1} \sum_{n \in \mathbb{Z}} L[f]\left(\sigma_{\left(1+\epsilon_{1}\right)}\right. \\
+n) s_{\left(1+\epsilon_{1}\right)}\left(t_{d}-n\right), \sum_{d=1}^{m} f\left(t_{d}\right) \\
\in \sum_{d=1}^{m} V\left(\varphi\left(t_{d}\right)\right) .
\end{gathered}
$$

(c) $\left\|\sum_{\epsilon_{1}=0}^{q}\left|Z_{\psi}\left(\sigma_{\left(1+\epsilon_{1}\right)}, \xi\right)\right|\right\|_{0}>0$.

Proof .Since

$$
\left\{L[f]\left(\sigma+\frac{1}{q-1} n\right): n \in \mathbb{Z}\right\}=\left\{L [ f ] \left(\sigma_{\left(1+\epsilon_{1}\right)}+\right.\right.
$$

$\left.n): 0 \leq \epsilon_{1} \leq q-1, n \in \mathbb{Z}\right\}$, we have a series of shifted symmetric multi-channel sampling for $q$ LTI systems $\quad\left\{L_{\left(1+\epsilon_{1}\right)}[\cdot]: 0 \leq \epsilon_{1} \leq q-1\right\} \quad$ with $L_{\left(1+\epsilon_{1}\right)}[\cdot]=L[\cdot], 0 \leq \epsilon_{1} \leq q-1$. Then

$$
g_{\left(1+\epsilon_{1}\right)}(\xi)=\frac{1}{2 \pi} Z_{\psi}\left(\sigma_{\left(1+\epsilon_{1}\right)}, \xi\right), 0 \leq \epsilon_{1} \leq q-1
$$

and

$$
G(\xi)^{*} G(\xi)=\frac{1}{(2 \pi)^{2}} \sum_{\epsilon_{1}=0}^{q-1}\left|Z_{\psi}\left(\sigma_{\left(1+\epsilon_{1}\right)}, \xi\right)\right|^{2}
$$

Hence $\quad \alpha_{G}>0$ if and only if $\left\|\sum_{\epsilon_{1}=0}^{q-1}\left|Z_{\psi}\left(\sigma_{\left(1+\epsilon_{1}\right)}, \xi\right)\right|\right\|_{0}>0$. Therefore, Corollary 3.7 is a consequence of Theorem 3.3.

\section{Example}

Let $\varphi_{0}=\sum_{d=1}^{m} \chi_{[0,1)}\left(t_{d}\right)$ be the Haar scaling function and

$$
\begin{aligned}
\sum_{d=1}^{m} \varphi_{1}\left(t_{d}\right)=\sum_{d=1}^{m} & \left(\varphi_{0} * \varphi_{0}\right)\left(t_{d}\right) \\
= & \sum_{d=1}^{m} \chi_{[0,1)}\left(t_{d}\right) \\
& +\sum_{d=1}^{m}\left(2-t_{d}\right) \chi_{[1,2)}\left(t_{d}\right)
\end{aligned}
$$

a B-spline of degree 1 . Then $\sum_{d=1}^{m} \varphi_{1}\left(t_{d}\right)$ is a continuous a series of Riesz generators [3], [22] and $\sup _{\mathbb{R}} \sum_{d=1}^{m} C_{\varphi_{1}}\left(t_{d}\right)=\sup _{\mathbb{R}} \sum_{n \in \mathbb{Z}} \sum_{d=1}^{m} \mid \varphi_{1}\left(t_{d}+\right.$ n) $/ 2<\infty$. First we take

$$
N=2, \sigma_{1}=\sigma_{2}=0,\left(1+\epsilon_{2}\right)_{1}=1,(1+
$$
$\epsilon 22=2$, and two LTI systems $L 1[\cdot]$ and $L 2[\cdot]$ with impulse responses $\sum_{d=1}^{m} l_{1}\left(t_{d}\right)=\sum_{d=1}^{m} \chi_{\left[\frac{-1}{2}, 0\right)}\left(t_{d}\right)$ and $\sum_{d=1}^{m} l_{2}\left(t_{d}\right)=\sum_{d=1}^{m} \chi_{\left[-1, \frac{-1}{2}\right)}\left(t_{d}\right)$. Then it's easy to see that

$$
\begin{gathered}
g_{1}(\xi)=\frac{1}{2 \pi} Z_{\psi_{1}}(0, \xi)=\frac{1}{2 \pi} \sum_{n \in \mathbb{R}} \psi_{1}(n) e^{-i n \xi} \\
=\frac{1}{16 \pi}\left(1+3 e^{-i \xi}\right)
\end{gathered}
$$




$$
\begin{gathered}
g_{2}(\xi)=\frac{1}{2 \pi} Z_{\psi_{2}}(0, \xi)=\frac{1}{2 \pi} \sum_{n \in \mathbb{R}} \psi_{2}(n) e^{-i n \xi} \\
=\frac{1}{16 \pi}\left(1+3 e^{-i \xi}\right),
\end{gathered}
$$

where

$$
\sum_{d=1}^{m} \psi_{\left(1+\epsilon_{1}\right)}\left(t_{d}\right)=\sum_{d=1}^{m} L_{\left(1+\epsilon_{1}\right)}[\varphi]\left(t_{d}\right) .
$$

Hence

$$
\begin{gathered}
g_{1,1}(\xi)=g_{1}(\xi), g_{1,2}(\xi)=g_{1}(\xi) e^{i \xi}, g_{2,1}(\xi) \\
=g_{2}(\xi)
\end{gathered}
$$

so that (see (4))

$$
\begin{aligned}
G(\xi)=\left[D g_{1,1}, D g_{1,2}, D g_{2,1}\right]^{T} & \\
= & \frac{1}{16 \pi}\left[\begin{array}{cc}
1+3 e^{-i \xi} & 1-3 e^{-i \xi} \\
3+e^{i \xi} & 3-e^{i \xi} \\
3+e^{-i \xi} & 3-e^{-i \xi}
\end{array}\right]
\end{aligned}
$$

And

$G(\xi) * G(\xi)=$

$\frac{1}{(16 \pi)^{2}}\left[\begin{array}{cc}30+18 \cos \xi & 8+6 i \sin \xi \\ 8-6 i \sin \xi & 30+18 \cos \xi\end{array}\right]$.

The eigenvalues of $G(\xi)^{*} G(\xi)$ are

$$
\frac{1}{(16 \pi)^{2}}\left[30+18 \cos \xi \pm \sqrt{100-36 \cos ^{2} \xi}\right]
$$

that

$$
\frac{1}{(16 \pi)^{2}} \leq \alpha_{G}=\left\|\lambda_{m}(\xi)\right\|_{0}<\beta_{G}=\left\|\lambda_{M}(\xi)\right\|_{\infty} \leq
$$

$\frac{58}{(16 \pi)^{2}}$.Hence by Theorem 3.3, there is a series of frames $\left\{\sum_{d=1}^{m} s_{\left(1+\epsilon_{1}\right)}\left(t_{d}-2 n\right): \epsilon_{1}=0,1,2,3\right.$ and $n \in \mathbb{Z}\}$ of the space of linear splines $\sum_{d=1}^{m} V\left(\varphi_{1}\left(t_{d}\right)\right)$ for which the following series of asymmetric multi-channel sampling expansions holds:

$$
\begin{aligned}
& \sum_{d=1}^{m} f\left(t_{d}\right)= \\
& \sum_{n \in \mathbb{Z}} \sum_{d=1}^{m}\left\{L_{1}[f](2 n) s_{1}\left(t_{d}-2 n\right)\right. \\
& \\
&+L_{1}[f](2 n+1) s_{2}\left(t_{d}-2 n\right) \\
&\left.+L_{2}[f](2 n) s_{3}\left(t_{d}-2 n\right)\right\},
\end{aligned}
$$

$$
f \in \sum_{d=1}^{m} V\left(\varphi_{1}\left(t_{d}\right)\right),
$$

which converges in $L^{2}(\mathbb{R})$ and absolutely and uniformly on $\mathbb{R}$.

We now take $N=1$ and $\sum_{d=1}^{m} l\left(t_{d}\right)=$ $\sum_{d=1}^{m} \delta\left(t_{d}\right)$ so that $L[\cdot]$ is the identity operator. Let $q(\geq 1)$ be an integer and $0 \leq \sigma<\frac{1}{q}$. Note first that for any fixed

$$
\begin{aligned}
\sum_{d=1}^{m} t_{d} \text { in } \mathbb{R}, \sum_{d=1}^{m} Z_{\varphi_{1}}\left(t_{d}, \xi\right) & \\
& =\sum_{n \in \mathbb{Z}} \sum_{d=1}^{m} \varphi_{1}\left(t_{d}+n\right) e^{-i n \xi} \\
& \in C[0,2 \pi]
\end{aligned}
$$

since $\sum_{d=1}^{m} \varphi_{1}\left(t_{d}\right)$ has compact support.Hence $\sum_{d=1}^{m}\left\|Z_{\varphi_{1}}\left(t_{d}, \cdot\right)\right\|_{L^{\infty}[0,2 \pi]}<\infty$ for each $\sum_{d=1}^{m} t_{d}$ in $\mathbb{R}$. Since $\quad Z_{\varphi_{1}}(\sigma, \xi)=\sigma+(1-\sigma) e^{-i \xi} \quad$ for $0 \leq \sigma<1,\left\|Z_{\varphi_{1}}(\sigma, \xi)\right\|_{0}=2\left|\sigma-\frac{1}{2}\right| \quad$ and $\left\|Z_{\varphi_{1}}(\sigma, \xi)\right\|_{\infty}=1$. Therefore, by Corollary 3.5 , for any $\sigma$ with $0 \leq \sigma<1$, there is a series of Riesz bases

$$
\left\{\sum_{d=1}^{m} s\left(t_{d}-n\right): n \in \mathbb{Z}\right\} \quad \text { of } \quad \sum_{d=1}^{m} V\left(\varphi\left(t_{d}\right)\right)
$$
such that

$$
\begin{gathered}
\sum_{d=1}^{m} f\left(t_{d}\right)=\sum_{n \in \mathbb{Z}} \sum_{d=1}^{m} f(\sigma+n) s\left(t_{d}-n\right), \sum_{d=1}^{m} f\left(t_{d}\right) \\
\in \sum_{d=1}^{m} V\left(\varphi_{1}\left(t_{d}\right)\right)
\end{gathered}
$$

if and only if $\sigma \neq \frac{1}{2}$. On the other hand, by Corollary 3.7, for any $q(\geq 2)$ and any $\sigma$ with $0 \leq \sigma<\frac{1}{q}$, there is a series of frames $\left\{\sum_{d=1}^{m} s_{\left(1+\epsilon_{1}\right)}\left(t_{d}-n\right): 0 \leq \epsilon_{1} \leq q-1, n \in \mathbb{Z}\right\}$ 
such that

$$
\begin{aligned}
\sum_{d=1}^{m} f\left(t_{d}\right)=\sum_{\epsilon_{1}=0}^{q-1} & \sum_{\in \mathbb{Z}} \sum_{d=1}^{m} f\left(\sigma+\frac{1}{q-1}\left(\epsilon_{1}\right)\right. \\
& +n) s_{\left(1+\epsilon_{1}\right)}\left(t_{d}-n\right), \sum_{d=1}^{m} f\left(t_{d}\right) \\
& \in \sum_{d=1}^{m} V\left(\varphi_{1}\left(t_{d}\right)\right) .
\end{aligned}
$$

As an example we show the following Corollary (see [22])

Corollary 3.7. Assume

$$
Z_{\psi}(2-\epsilon, \xi) \in L^{\infty}[0,2 \pi], 0 \leq \epsilon_{1} \leq q-1
$$

then the following are all equivalent.

(a) There is a series of frames $\left\{\sum_{d=1}^{m} s_{n}\left(t_{d}\right): n \in\right.$ $\mathbb{Z}\}$ of $\sum_{d=1}^{m} V\left(\varphi\left(t_{d}\right)\right)$ for which

$$
\begin{gathered}
\sum_{d=1}^{m} f\left(t_{d}\right)=\sum_{n \in \mathbb{Z}} \sum_{d=1}^{m} L[f](2-\epsilon) s_{n}\left(t_{d}\right), \sum_{d=1}^{m} f\left(t_{d}\right) \\
\in \sum_{d=1}^{m} V\left(\varphi\left(t_{d}\right)\right) .
\end{gathered}
$$

(b) There is a series of frames

$$
\left\{\sum_{d=1}^{m} s_{\left(1+\epsilon_{1}\right)}\left(t_{d}-n\right): \epsilon_{1}>0, n \in \mathbb{Z}\right\}
$$

of

$$
\sum_{d=1}^{m} V\left(\varphi\left(t_{d}\right)\right)
$$

for which

$$
\begin{aligned}
& \sum_{d=1}^{m} f\left(t_{d}\right)=\sum_{n \in \mathbb{Z}} \sum_{\epsilon_{1} \geq 0} \sum_{d=1}^{m} L[f](n-\epsilon) s_{\left(1+\epsilon_{1}\right)}\left(t_{d}\right. \\
& -n), \sum_{d=1}^{m} f\left(t_{d}\right) \in \sum_{d=1}^{m} V\left(\varphi\left(t_{d}\right)\right) . \\
& \text { (c) }\left\|\sum_{\epsilon_{1} \geq 0}\left|Z_{\psi}(2-\epsilon, \xi)\right|\right\|_{0}>0 .
\end{aligned}
$$

\section{Proof .Since}

$\{L[f](2-\epsilon)\}=\{L[f](n-\epsilon): n \in \mathbb{Z}\} . \quad$ Now we have $\left\{L_{\left(1+\epsilon_{1}\right)}[\cdot]: \epsilon_{1}>0\right\}$ with

$$
L_{\left(1+\epsilon_{1}\right)}[\cdot]=L[\cdot], \epsilon_{1}>0 \text {. Then } g_{\left(1+\epsilon_{1}\right)}(\xi)=
$$

$\frac{1}{2 \pi} Z_{\psi}(2-\epsilon, \xi), \epsilon_{1}>0 \quad$ and $\quad G(\xi)^{*} G(\xi)=$ $\frac{1}{(2 \pi)^{2}} \sum_{\epsilon_{1} \geq 0}\left|Z_{\psi}(2-\epsilon, \xi)\right|^{2}$. There for $\alpha_{G}>0$ if and only if

$$
\left\|\sum_{\epsilon_{1} \geq 0}\left|Z_{\psi}(2-\epsilon, \xi)\right|\right\|_{0}>0 .
$$

\section{References}

[1] K.F. Cheung, A multi-dimensional extension of Papoulis's generalized sampling expansion with application in minimum density sampling, in:R.J. Marks II (Ed.), Advanced Topics in Shannon Sampling and Interpolation Theory, Springer-Verlag, Berlin, 1993.

[2] O. Christensen, An Introduction to Frames and Riesz Bases, Birkhäuser, Boston, 2001.

[3] C.K. Chui, An Introduction to Wavelets, Academic Press, New York, 1992.

[4] I. Djokovic, P.P. Vaidyanathan, Generalized sampling theorems in multiresolution subspaces, IEEE Trans. Signal Process. 45 (1997) 583-599.

[5] T. Eldar, A.V. Oppenheim, Filterbank reconstruction of bandlimited signals from nonuniform and generalized samples, IEEE Trans. Signal Process. 48(2000) 2864-2875.

[6] A. Feuer, On the necessity of Papoulis' result for multi-dimensional GSE, IEEE Signal Process. Lett. 11 (2004) 420-422.

[7] L.J. Fogel, A note on the sampling theorem, IRE Trans. Inform. Theory IT-1(1995) 47-48.

[8] A.G. Garcia, M.A. Hernundez-Medina, G. Pérez-Villalón, Oversampling and reconstruction functions with compact support, J. Comput. Appl. Math. 227(2009) 245-253.

[9] A.G. Garcia, G. Pérez-Villalón, Dual frames in $L^{2}(0,1)$ connected with generalized sampling in shift-invariant spaces, Appl. Comput. Harmon. Anal. 20(2006) 422-433.

[10] A.G. Garcia, G. Pérez-Villalón, A. Portal, Riesz bases in $L^{2}(0,1)$ related to sampling in shift-invariant spaces, J. Math. Anal. Appl. 308 (2005) 703-713.

[11] Y.M. Hong, J.M. Kim, K.H. Kwon, E.H. Lee, Channeled sampling in shift invariant spaces, Int. J. Wavelets Multiresolut. Inf. Process. 5 (2007) 753-767. 
[12] A.J.E.M. Janssen, The Zak transform and sampling theorems for wavelet subspaces, IEEE Trans. Signal Process. 41 (1993) 3360-3364.

[13] J.M. Kim, K.H. Kwon, Sampling expansion in shift invariant spaces, Int. J. Wavelets Multiresolut. Inf. Process. 6 (2008) 223-248.

[14] J.M. Kim, K.H. Kwon, Vector sampling expansion in Riesz bases setting and its aliasing error, Appl. Comput. Harmon. Anal. 25 (2008) 315-334.

[15] G.E.C. Nogueira, A. Ferreira, Higher order sampling and recovering of lowpass signals, IEEE Trans. Signal Process. 48 (2000) 2169-2171.

[16] A. Papoulis, Generalized sampling expansion, IEEE Trans. Circuits Syst. 24 (1977) 652-654.

[17] D. Seidner, M. Feder, Vector sampling expansion, IEEE
Trans. Signal Process. 48 (2000) 401-1406.

[18] C.E. Shannon, Communication in the presence of noise, Proc. Inst. Radio Eng. 37 (1949) 10-21.

[19] M. Unser, J. Zerubia, Generalized sampling: Stability and performance analysis, IEEE Trans. Signal Process. 45 (1997) 2941-2950.

[20] M. Unser, J. Zerubia, A generalized sampling theory without band-limiting constraints, IEEE Trans. Circuits Syst. II 45 (1998) 959-969.

[21] X. Zhou, W. Sun, On the sampling theorem for wavelet subspaces, J. Fourier Anal. Appl. 5 (1999) 347-354.

[22] S. Kang , J.M. Kim, K.H. Kwon Asymmetric multi-channel sampling in shift invariant spaces J. Math. Anal. Appl. 367 (2010) 20-28 